\title{
Large-area InP-based crystalline nanomembrane flexible photodetectors
}

\author{
Weiquan Yang, ${ }^{1}$ Hongjun Yang, ${ }^{1}$ Guoxuan Qin, ${ }^{2}$ Zhenqiang $M a,{ }^{2}$ Jesper Berggren, ${ }^{3}$ \\ Mattias Hammar, ${ }^{3}$ Richard Soref, ${ }^{4}$ and Weidong Zhou ${ }^{1, a)}$ \\ ${ }^{1}$ Department of Electrical Engineering, NanoFAB Center, University of Texas at Arlington, Arlington, \\ Texas 76019-0016, USA \\ ${ }^{2}$ Department of Electrical and Computer Engineering, University of Wisconsin-Madison, \\ Wisconsin 53706, USA \\ ${ }^{3}$ School of Information and Communication Technology (ICT), The Royal Institute of Technology (KTH), \\ 16440 Kista, Sweden \\ ${ }^{4}$ Sensors Directorate, Air Force Research Laboratory, AFRL/RYHC, Hanscom Air Force Base, \\ Massachusetts 01731-2909, USA
}

(Received 29 January 2010; accepted 4 March 2010; published online 25 March 2010)

\begin{abstract}
Large-area $\left(3 \times 3 \mathrm{~mm}^{2}\right)$ flexible photodetectors were realized, based on crystalline InP semiconductor nanomembranes transferred to flexible polyethylene terephthalate substrates. Very low dark current (a few microamperes) and high responsivity $(0.12 \mathrm{~A} / \mathrm{W})$ were demonstrated for flexible InP p-i-n photodetectors. Bending characteristics were also investigated for this type of flexible crystalline semiconductor photodetector, and it was found that, whereas the dark current was independent of bending radii, the photocurrent degraded, depending on the bending radii. (C) 2010 American Institute of Physics. [doi:10.1063/1.3372635]
\end{abstract}

Flexible electronic and photonic structures consisting of electronic and photonic devices that can be bent, expanded, and manipulated are of great scientific and engineering importance. Such devices find their applications ranging from flexible imaging/displays, sensors, solar cells, and conformal electronic/photonic integrated systems to potential integration into artificial muscles or biological tissues. Most flexible photonics research so far is based on organic, polymer, and/or amorphous semiconductor material systems. Crystalline semiconductor nanomembranes (NMs) offer unprecedented opportunities for high performance flexible photonics. High quality single crystalline silicon NMs (SiNMs) have been transferred onto various foreign substrates, such as glass, flexible polyethylene terephthalate (PET) plastics, etc., based on low temperature transfer and stacking processes. ${ }^{1-5}$ Very high performance electronics based on transferable $\mathrm{Si} /$ SiGe NMs were already reported. ${ }^{1-3}$ Flexible Ge photodetectors (PDs) were also reported recently. ${ }^{6}$ We have also reported various photonic devices based on Fano resonances on $\mathrm{Si}$, glass, and flexible PET substrates. ${ }^{7-9}$ In addition to Group IV materials ( $\mathrm{Si}, \mathrm{Ge}$, etc.), NMs based on III-V (GaAs, InP, etc.) and other material systems are also being developed for heterogeneous integration (membrane stacking) on $\mathrm{Si}$ and other foreign substrates, with desired electronic and photonic functions. ${ }^{10,11}$

However, two significant challenges remain in realizing practical large-area photonic devices based on stacked crystalline semiconductor NMs. One of them is reliable transfer of large-area crystalline semiconductor NMs, especially for those fragile materials systems (e.g., GaAs and InP). The second challenge is the incorporation of metal contacts for the desired electrical properties of photonic devices. To address these two challenges, we propose and report here a frame-assisted membrane transfer (FAMT) process. Based on this process, we have transferred InP NMs onto flexible PET substrates, and have demonstrated large area flexible

${ }^{a)}$ Electronic mail: wzhou@uta.edu.
PDs. The characteristics of flexible InP PDs are also reported here.

Shown in Fig. 1 is the process flow of the FAMT process. The starting material consists of the device layers (InP $\mathrm{p}$-i-n structure, in this case) grown on top of a sacrificial layer (e.g., InGaAs for the InP material system). ${ }^{1,9}$ As shown in Fig. 1(a), release holes were formed on the top device layer, based on a wet or dry etching process. These release holes are formed to facilitate the selective wet-etching of sacrificial layer beneath the device layer. Before the actual selective wet-etch step for the release of the top device layer, a metal frame [Fig. 1(b)] is formed on top of the device layer. This metal frame layer serves as the supporting frame to increase the mechanical strength of the to-be released InP NMs. At the same time, it can also serve as the top finger contact layer for the devices to be built on this transferred NM. After selective wet-etching of the sacrificial layer, the top device layer is released from the hosting substrate [Fig. 1(c)] and transferred to a foreign substrate [e.g., PET substrate, Fig. 1(d)]. Based on this process, we have been able to transfer different sizes of InP NMs, up to $3 \times 3 \mathrm{~mm}^{2}$, solely limited by the feature size of the mask used.

Large-area flexible PDs were demonstrated, based on the crystalline InP p-i-n NMs transferred to a flexible PET sub-

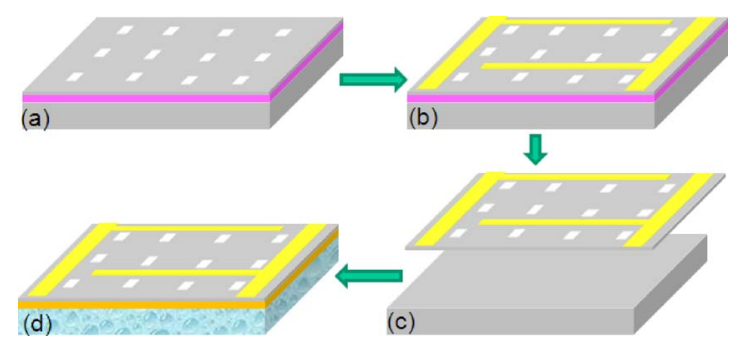

FIG. 1. (Color online) A flow chart for FAMT process. (a) Formation of release holes on the top device layer; (b) formation of metal-frames (finger contacts) on top of the device layer; (c) release of the device layer; and (d) transfer of the device layer onto flexible PET substrate or other foreign substrates. 


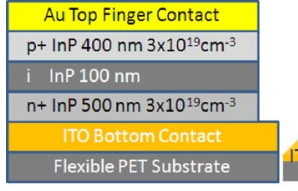

(a)

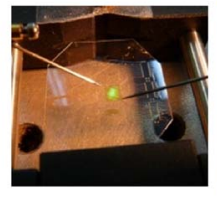

(c)

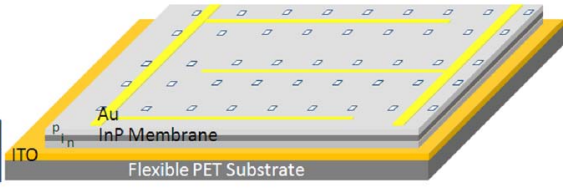

(b)

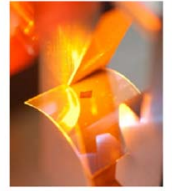

(d)

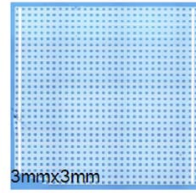

(e)

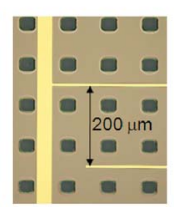

(f)
FIG. 2. (Color online) (a) Cross-sectional, and (b) three-dimensional views of flexible InP p-i-n NM PD on ITO/PET substrate. (c) A micrograph of fabricated flexible InP PD under test. [(d)-(f)] Zoom-in views of a fabricated large area $\left(3 \times 3 \mathrm{~mm}^{2}\right)$ InP PD on flexible PET substrate.

strate as shown schematically in Figs. 2(a) and 2(b). The starting material is a $\mathrm{p}-\mathrm{i}-\mathrm{n}$ InP layer (total thickness of $1 \mu \mathrm{m})$ grown on top of an InP substrate, with an InGaAs sacrificial layer sandwiched in between. The thicknesses for the p-, i-, and n-type InP layers are $400 \mathrm{~nm}, 100 \mathrm{~nm}$, and $500 \mathrm{~nm}$, respectively. First, release holes were formed on the top InP layer, based on selective wet etching of InP layer with a mixed solution of hydrochloric acid and phosphoric acid (1:4). Second, an Au finger contact (90 nm thick) was formed on top of the p-InP layer. Third, the InGaAs layer was then selectively etched away by immersing the sample in a $\mathrm{HF}: \mathrm{H}_{2} \mathrm{O}_{2}: \mathrm{H}_{2} \mathrm{O}(1: 1: 10)$ solution, and the metal framed InP layer detached from the InP host substrate. Finally, InP NM was transferred to the indium tin oxide (ITO)/PET sub-

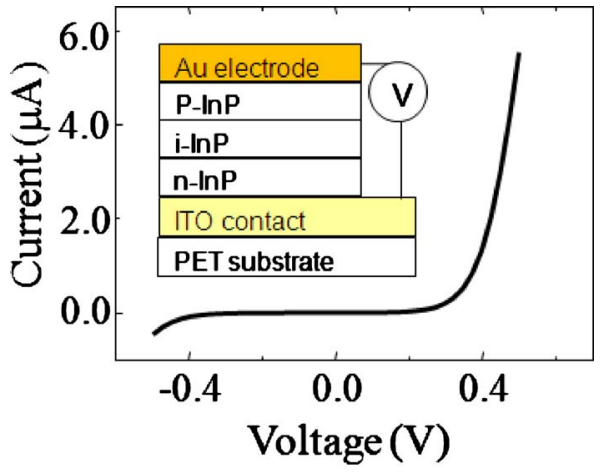

(a)

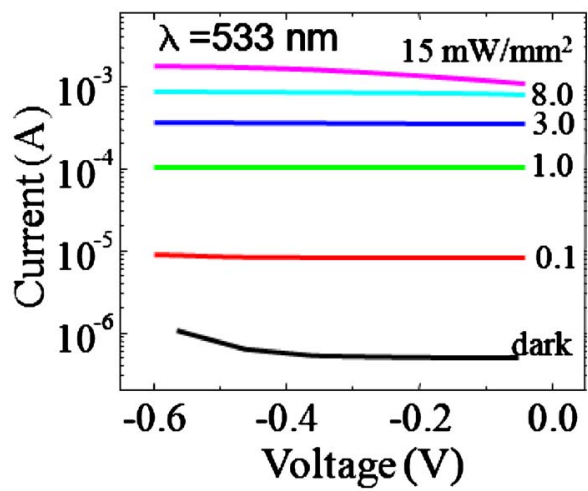

(b)

FIG. 3. (Color online) Measured flexible InP p-i-n PD characteristics. (a) Measured dark current-voltage characteristics. (b) Measured photocurrents at different incident optical powers for a $533 \mathrm{~nm}$ wavelength light source.

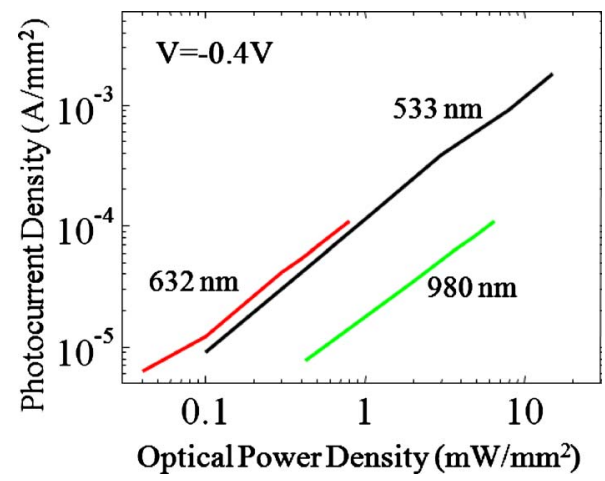

FIG. 4. (Color online) Measured photocurrent density for different incident optical power levels with three different wavelengths, at reverse bias of $-0.4 \mathrm{~V}$.

strate. A complete flexible InP PD array was formed, with top Au finger contact and bottom ITO transparent contact. A micrograph of an actual device under test is shown in Fig. 2(c). Shown in Figs. 2(d)-2(f) are device micrographs with different zoom-in scales.

The measured flexible InP p-i-n PD characteristics are shown in Figs. 3-5. Based on the simple stacked bottom ITO contact, very low dark current of less than $1 \mu \mathrm{A}$ was observed at reverse biases up to $-0.5 \mathrm{~V}$ [Fig. 3(a)]. Shown in Fig. 3(b) are the photocurrents measured at different incident optical powers, with an incident light source of $533 \mathrm{~nm}$ wavelength. A very large operation window is feasible due to the linear response. As shown in Fig. 4 for light sources with three different wavelengths $(533,632$, and $980 \mathrm{~nm})$, very

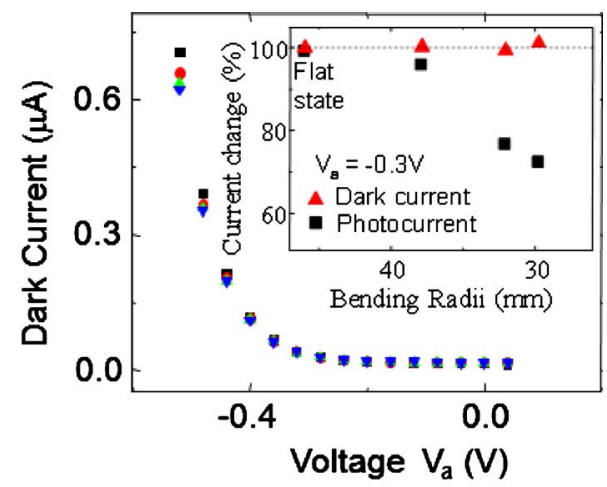

(a)

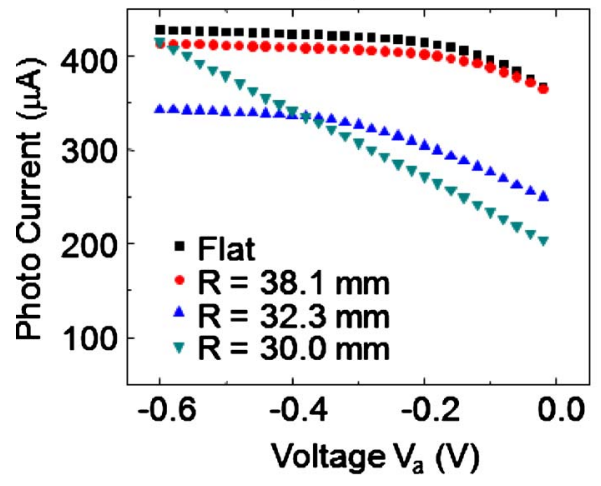

(b)

FIG. 5. (Color online) Measured bending characteristics for a fabricated flexible InP PD at different bending radii. (a) Dark currents; and (b) photocurrents. The relative changes in measured dark and photocurrent are also shown in the inset of (a), at a bias voltage of $-0.3 \mathrm{~V}$. 
linear photoresponse was observed for the power ranges measured. The measured quantum efficiencies are similar at the incident wavelengths of 533 and $632 \mathrm{~nm}$ but are significantly reduced at $980 \mathrm{~nm}$, mostly due to the reduced absorption near the absorption edge of InP.

The measured responsivity of the flexible InP p-i-n PD is $0.12 \mathrm{~A} / \mathrm{W}$ with incident light of $533 \mathrm{~nm}$ wavelength. Based on the absorption coefficient value of $10^{5} \mathrm{~cm}^{-1}$ for InP at $533 \mathrm{~nm}$, we have derived the theoretical responsivity for the $1 \mu \mathrm{m}$ thick InP PD to be about 0.29 A/W. The measurement results agree very well with the theoretical predictions.

The bending characteristics are shown in Fig. 5 for the flexible InP p-i-n PD with incident light wavelength of 533 $\mathrm{nm}$ and the optical incident power intensity of $3 \mathrm{~mW} / \mathrm{mm}^{2}$. For different bending radii, the dark current does not change much [Fig. 5(a)]. On the other hand, as shown in Fig. 5(b), the photocurrent initially changed only slightly when large bending radii were employed (down to $38.1 \mathrm{~mm}$ ). However, when the PD was bent further, the photocurrent decreased quickly. The relative photocurrent reduction is also plotted in the inset of Fig. 5(a), for a bias voltage of $-0.3 \mathrm{~V}$, where up to $71 \%$ reduction was observed for the bending radius of $30 \mathrm{~mm}$. This change in PD performance maybe associated with the nonuniform illumination from the light source used for the test, as well as the possible strain-induced absorption reduction. These results indicate that the flexible InP PD will work when the bending radius is larger than $38.1 \mathrm{~mm}$. However, it is feasible to further reduce the bending radius without much degradation in PD performance. $4,6,11$

In summary, we report here very large flexible PDs, based on a FAMT process for the transfer of a very soft crystalline semiconductor NM onto flexible substrate. High performance flexible InP $\mathrm{p}-\mathrm{i}-\mathrm{n}$ PDs were demonstrated with responsivity of $0.12 \mathrm{~A} / \mathrm{W}$ at $533 \mathrm{~nm}$ for $1 \mu \mathrm{m}$ thick $\mathrm{InP}$ NMs. The flexible InP p-i-n PDs perform well when the bending radius is larger than $38.1 \mathrm{~mm}$.

W.Y. appreciates the help from Dr. Zexuan Qiang and Santhad Chuwongin on detector testing. This work is supported in part by U.S. AFOSR MURI program under Grant No. FA9550-08-1-0337, by U.S. ARO under Grant No. W911NF-09-1-0505, and by U.S. AFRL under Grant No. FA 8650-07-2-5061.

${ }^{1}$ H. C. Yuan, Z. Ma, M. M. Roberts, D. E. Savage, and M. G. Lagally, J. Appl. Phys. 100, 013708 (2006)

${ }^{2}$ H. C. Yuan and Z. Ma, Appl. Phys. Lett. 89, 212105 (2006).

${ }^{3}$ H. C. Yuan, G. K. Celler, and Z. Ma, J. Appl. Phys. 102, 034501 (2007).

${ }^{4}$ S. A. Scott and M. G. Lagally, J. Phys. D: Appl. Phys. 40, R75 (2007).

${ }^{5}$ J. A. Rogers, Z. Bao, K. Baldwin, A. Dodabalapur, B. Crone, V. R. Raju, V. Kuck, H. Katz, K. Amundson, and J. Ewing, Proc. Natl. Acad. Sci. U.S.A. 98, 4835 (2001).

${ }^{6}$ H. Yuan, J. Shin, G. Qin, L. Sun, P. Bhattacharya, M. G. Lagally, G. K. Celler, and Z. Ma, Appl. Phys. Lett. 94, 013102 (2009).

${ }^{7}$ H. Yang, Z. Qiang, H. Pang, Z. Ma, and W. D. Zhou, Electron. Lett. 44, 858 (2008).

${ }^{8}$ Z. Qiang, H. Yang, L. Chen, H. Pang, Z. Ma, and W. Zhou, Appl. Phys. Lett. 93, 061106 (2008).

${ }^{9}$ L. Chen, Z. Qiang, H. Yang, H. Pang, Z. Ma, and W. D. Zhou, Opt. Express 17, 8396 (2009).

${ }^{10}$ K. Lee, J. Lee, H. Hwang, Z. Reitmeier, R. Davis, J. A. Rogers, and R. Nuzzo, Small 1, 1164 (2005).

${ }^{11}$ S.-I. Park, Y. Xiong, R.-H. Kim, P. Elvikis, M. Meitl, D.-H. Kim, J. Wu, J. Yoon, C.-J. Yu, Z. Liu, Y. Huang, K.-C. Hwang, P. Ferreira, X. Li, K. Choquette, and J. A. Rogers, Science 325, 977 (2009). 